\title{
Macrophages related to Bruch's Membrane in Age- Related Macular Degeneration
}

\author{
M. C. KILLINGSWORTH, J. P. SARKS and S. H. SARKS \\ Sydney, Australia
}

\begin{abstract}
Summary
This report describes focal thinning of Bruch's membrane in age-related macular degeneration and suggests a role for macrophages in this process. Electron microscopy was performed on four eyes ranging from normal ageing to age-related macular degeneration with early subretinal neovascularisation. As degeneration progressed multiple segments of thinning of Bruch's membrane were observed and macrophages appeared to engulf fragments of the outer collagenous zone. An associated finding at this stage was the shedding of membranous debris by the retinal pigment epithelium and its deposition within Bruch's membrane. This debris forms the basis of soft drusen but in the presence of this material thinning of Bruch's membrane was also observed beneath hard drusen. It is suggested that the widespread accumulation of phospholipid-containing membranes together with the focal concentration of lipid in hard drusen attract macrophages.
\end{abstract}

It has previously been proposed from morphological observations that subretinal neovascularisation (SRN) in age-related macular degeneration (AMD) may be a reaction to the products of cellular degeneration, ${ }^{1}$ notably from the retinal pigment epithelium (RPE). Since macrophages are attracted to debris, the aim of this study was to present evidence supporting the role of macrophages in establishing a passage through Bruch's membrane and to note the nature of the debris present, with particular reference to drusen.

As AMD develops, chronic inflammatory cells are found in relation to the outer surface of Bruch's membrane. ${ }^{2}$ However, fibroblasts, lymphocytes and macrophages may also occur at the site of narrowed or obliterated choroidal capillaries. ${ }^{3,4}$ This report is therefore limited to eyes demonstrating an associated focal thinning of Bruch's membrane.

\section{Materal and Methods}

The eyes reported herein have been exam- ined as part of an ongoing clinicopathological study of elderly institutionalised patients and were classified accordingly. ${ }^{5}$ The onset of AMD had been characterised by the presence of a thin continuous basal linear deposit (BLD) external to the RPE at the macula, since most such eyes had shown some reduction in visual acuity. Ultrastructural findings are described in four eyes representing normal ageing, early degeneration, more advanced degeneration, and degeneration with early choroidal neovascularisation respectively.

The eyes were prepared for electron microscopy using techniques previously described. ${ }^{2}$ Initially semithin sections of $0.5 \mu$ were examined under the light microscope. In areas where cellular activity was present, ultrathin sections were cut and examined under a Zeiss EM10CR electron microscope.

In this report Bruch's membrane is regarded as the sheetlike condensation of the innermost portion of the choridal stroma, 


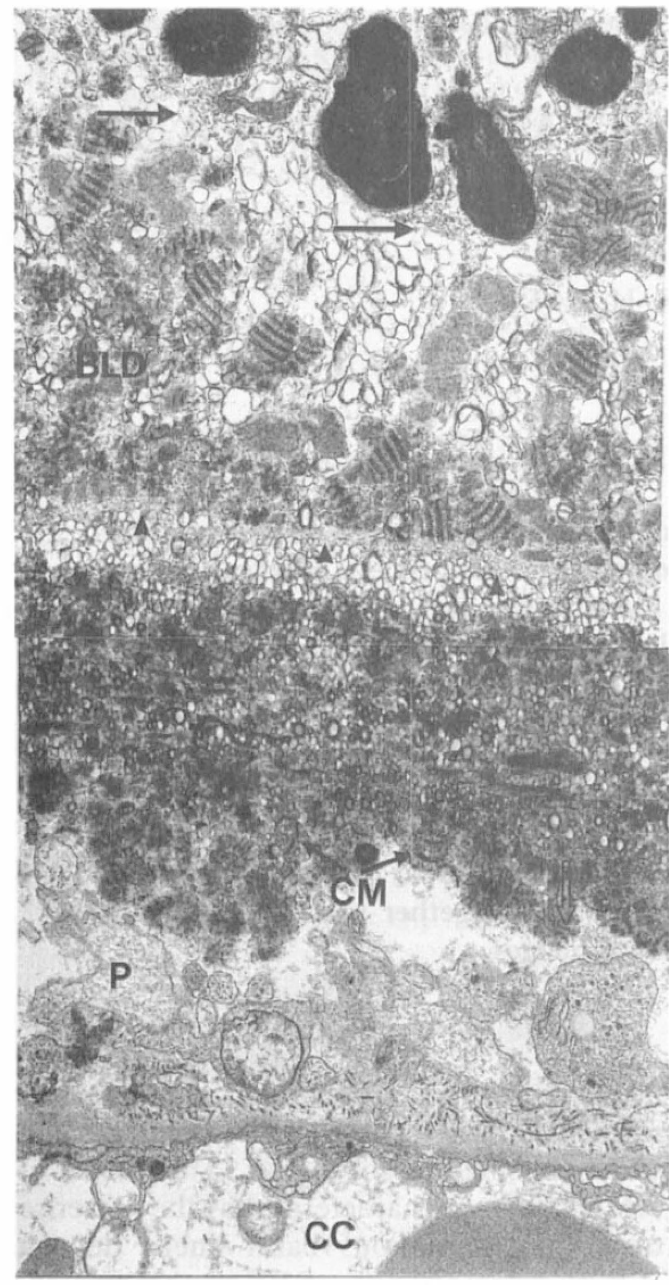

Fig. 1. Electron micrograph illustrating changes developing between the RPE and the choriocapillaris $(C C)$ in $A M D$. The basal laminar deposit (BLD) consists of banded, fibrillar and homogeneous material. The basal plasma membrane of the RPE (horizontal black arrows) appears to be shedding coiled membranous debris which passes through the basement membrane (arrowheads) to form several layers on its external aspect. Some can then be traced into Bruch's membrane which is thickened and dense. Fibrous banded material (open arrow) and fragments of coated membrane $(C M)$ can be identified. Cell process $(P)$ lies between Bruch's membrane and choroidal capillary. Eye belonged to an 81-year-old man noted to have small soft drusen and vision of $6 / 6 p$ one year before death. $\times 12,040$

consisting of a layer of collagen on either side of a layer of elastic tissue but excluding the basal laminas of the RPE and choroidal capillary endothelium, as defined by Gass. ${ }^{6}$

\section{Results}

Figure 1 illustrates the two extracellular materials consistently found under the RPE at the fovea once AMD has commenced. The BLD shown consists of banded, fibrillar and homogeneous material continuous with the basement membrane of the RPE. This layer formed the basis of the previous histological classification $^{5}$ as its thickness remained relatively constant between eyes at a similar stage of degeneration.

Greater variation occurred in the quantity of coiled membranous debris that had the structure of a lipid bilayer. This material lay in close contact with the basal plasma membrane of the RPE and also formed a continuous stratum external to the basement membrane. Smaller coils could be traced into Bruch's membrane as far as the outer collagenous zone. In addition, the thickened Bruch's membrane contained fibrous banded material and fragments of coated membrane. At this stage cell processes may be found related to the outer collagenous zone.

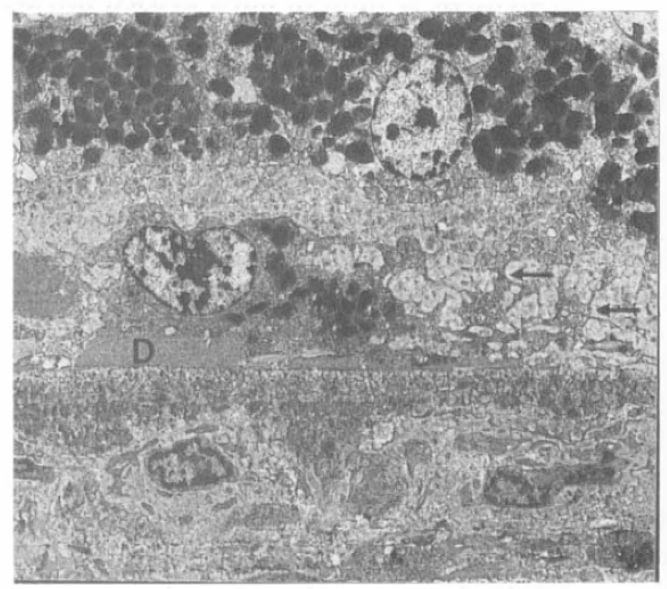

Fig. 2. Case 1 demonstrating cellular activity in normal ageing. Cell on Bruch's membrane is closely related to amorphous material (D) of a small hard druse. Cell displays numerous cell processes continuous with overlying RPE (arrows), melanolipofuscin granules and a nucleus resembling that of an RPE cell. Cells have replaced underlying choriocapillaris but no communication through Bruch's membrane was found. The only fundus abnormality noted had been a parafoveal pigment clump associated with a cluster of small hard drusen. Little change developed over a six-year period of observation prior to the patient's death at age 67 and vision remained $6 / 6 . \times 3,470$ 


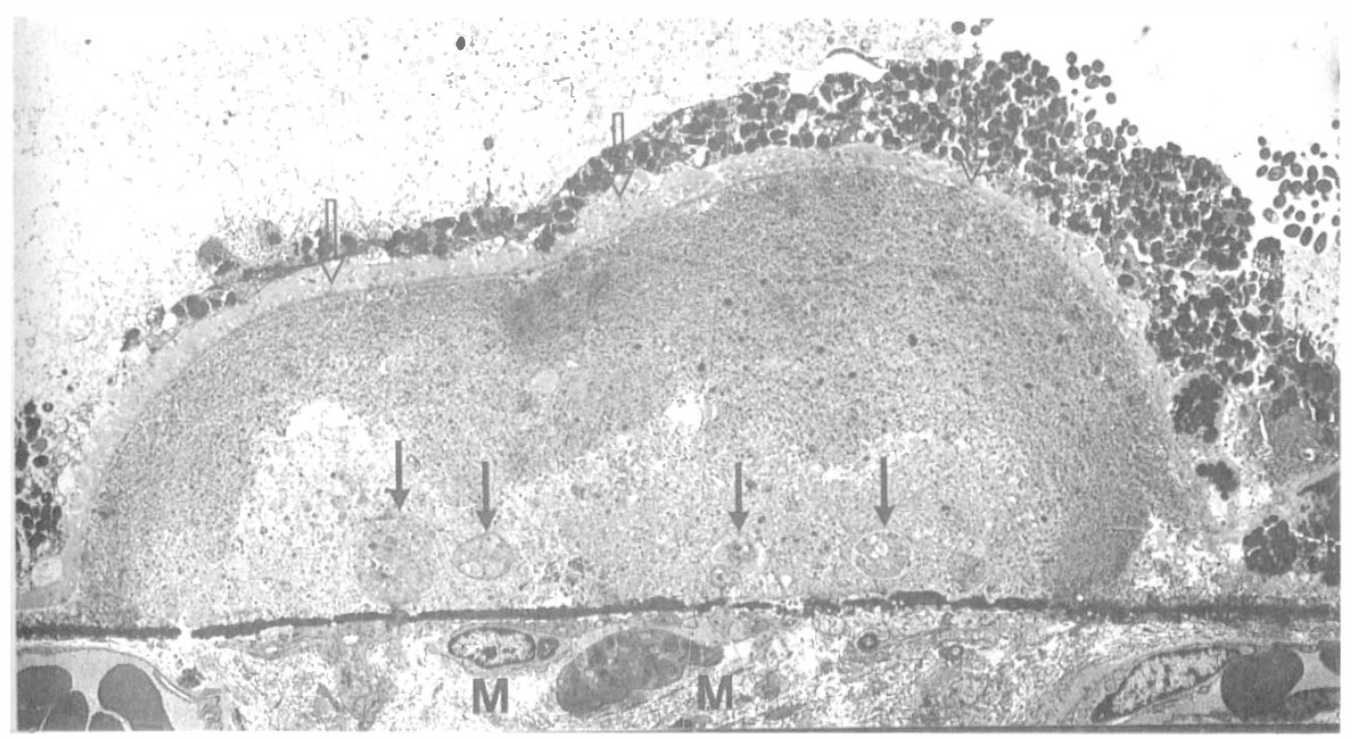

Fig. 3. (Case 2) Section through a large hard druse from an eye of a 72-year-old man with early degeneration and 6/9 vision. Druse is covered by continuous BLD (open arrows). Druse contents appear to be breaking down. Distended cell processes (black arrows) in druse belong to macrophages (M) lying external to Bruch's membrane. Only the elastic layer of Bruch's membrane remains. $\times 2,300$

\section{Case 1. Normal ageing}

An example of normal ageing was an eye in which the fundus had shown a small parafoveal pigment clump related to a cluster of small hard drusen, but which was otherwise normal. The BLD and membranous debris were absent. A cell with numerous processes was noted on the inner surface of Bruch's membrane, apparently engaged in the removal of a hard druse (Fig 2). Features suggest- ing this cell may have been derived from the RPE were the presence of melanolipofuscin granules, abundant rough endoplasmic reticulum, cytoplasmic processes continuous with overlying cells and the failure to demonstrate a communication with the choroid.

\section{Case 2. Early AMD with hard drusen.}

Figures 3 and 4 illustrate an equatorial hard

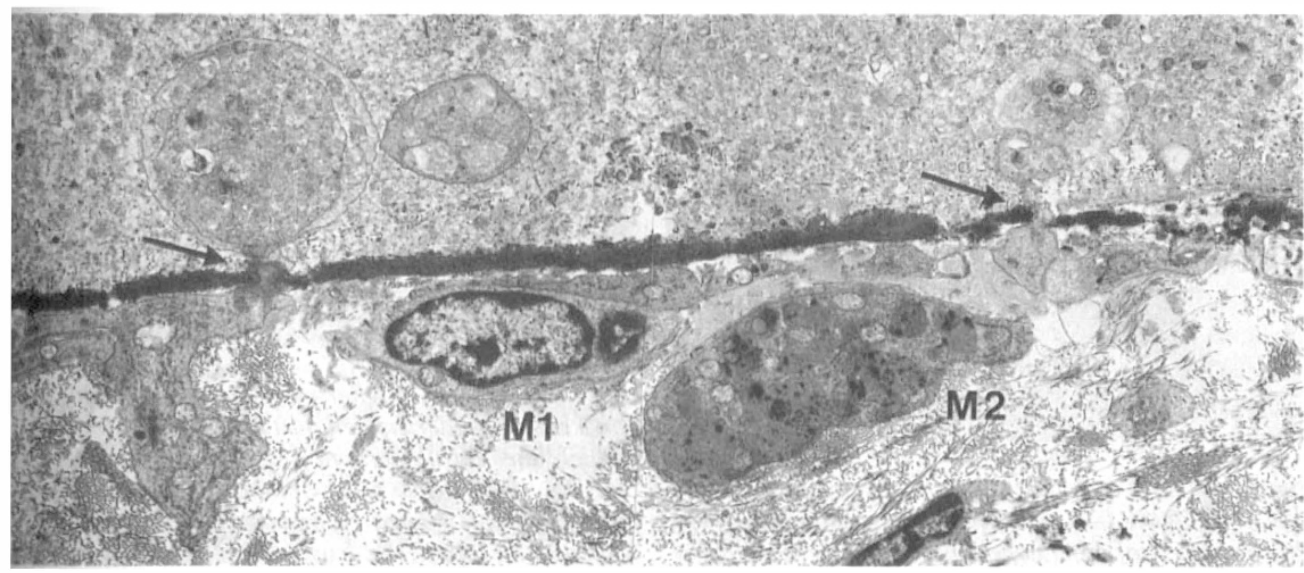

Fig. 4. (Case 2) Higher magnification of cell processes passing through small gaps in elastic lamina shown in Figure 3 (arrows). The processes expand within the druse and appear to contain degraded cytoplasm. $M 1=$ bilobed nucleus of a macrophage. M2-Macrophage containing heterogeneous secondary lysosomes. $\times 6,300$ 


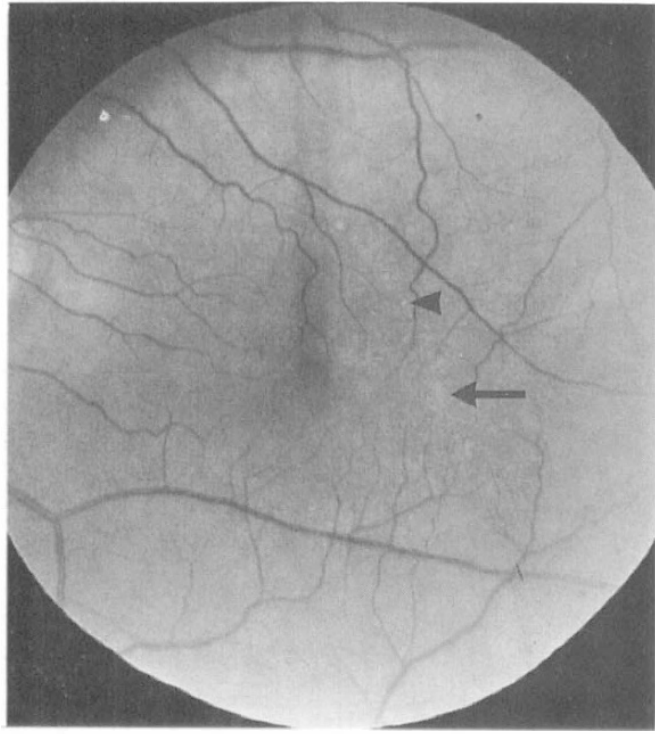

Fig. 5. (Case 3) Left eye of an 82-year-old man with 6/6 vision, demonstrating small hard drusen (arrowhead) and shallow soft drusen (arrow). There is minimal disturbance of retinal pigmentation.

druse from an eye of a 72-year old man in whom clinical examination had shown early degeneration with $6 / 9$ vision. The collagenous zones of Bruch's membrane were no longer

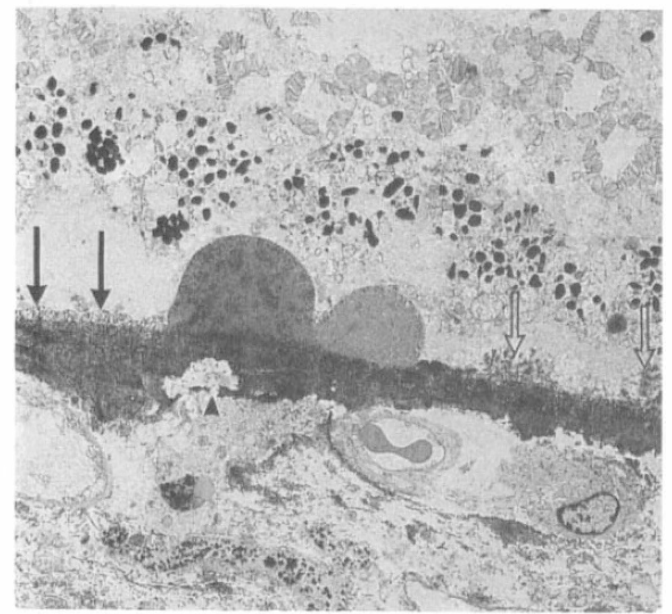

Fig. 6. (Case 3) A pair of hard drusen from the eye shown in Figure 5. Bruch's membrane is thickened but shows erosion of outer collagenous zone beneath druse (arrowhead). Other changes noted are a thin layer of membranous debris lying external to the basement membrane (black arrows) and clumps of early-type $B L D$ (open arrows). $\times 2,350$ evident and only the elastic lamina remained. The large irregular cells lying beneath the druse were considered to be macrophages on the grounds of having bilobed nuclei, abundant lysosomal granules, a well-organised cytoskeleton and prominent residual bodies. Several cell processes passed into the druse where they expanded into blebs of degenerating cytoplasm. The contents of the druse in the vicinity of these processes appeared to be breaking down.

Case 3. AMD with mainly small hard drusen but also soft drusen

(Figure 5). In this eye from an 82-year-old man, macrophages applied to the outer surface of Bruch's membrane were widespread. Although the BLD and a continuous layer of membranous debris were also present, thinning of the outer collagenous zone commenced beneath small hard drusen (Figures 6 \& 7).

Beneath one druse Bruch's membrane had almost disappeared except for a tenuous elastic layer and a choroidal capillary had

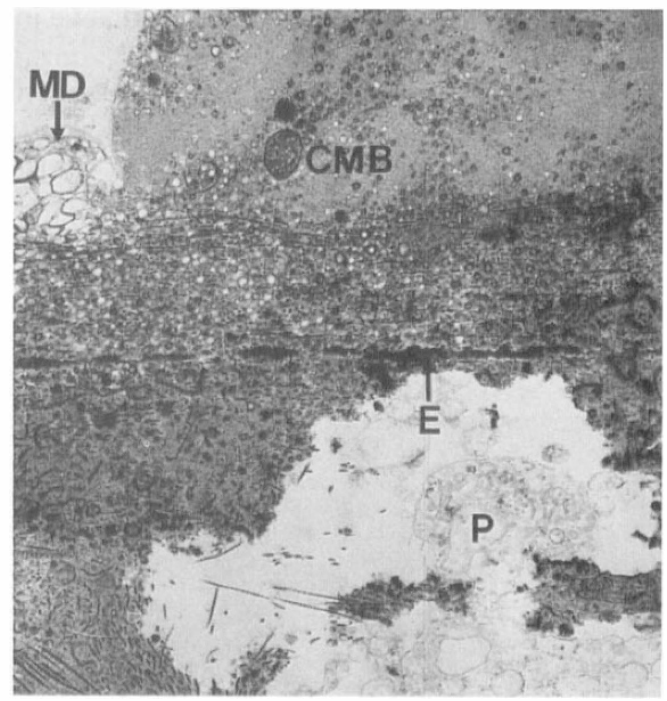

Fig. 7. (Case 3) Higher magnification showing a cell process $(P)$ under eroded part of Bruch's membrane in which the elastic lamina $(E)$ is exposed. Adjacent membrane is dense and contains fragments of coated membrane. A coated membrane-bound body (CBM) can also be seen in the druse. Cellular activity related to the outer surface of Bruch's membrane was not evident until a layer of membranous debris (MD) was also present on the internal surface of Bruch's membrane. $\times$ 12,040 


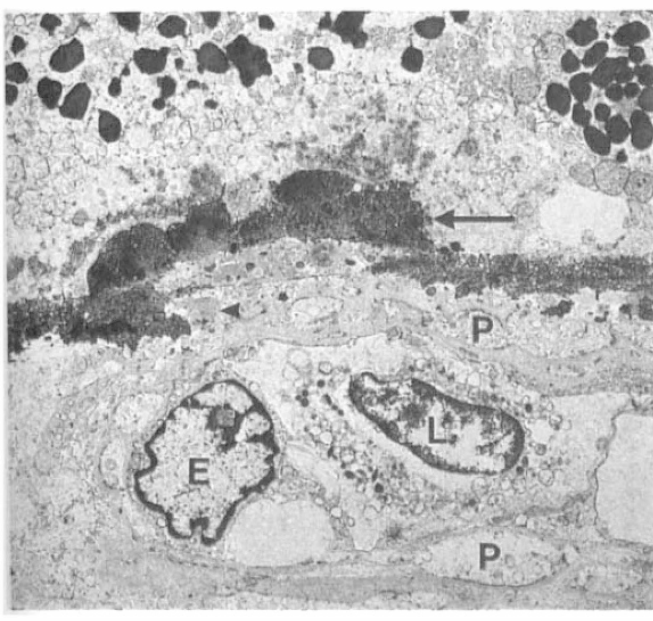

Fig. 8. (Case 3) Another hard druse (arrow) containing electron dense bodies. The druse and Bruch's membrane have been eroded by a cell process (arrowhead) and only a tenuous elastic layer remains. A choroidal capillary with a thick basement membrane lies immediately beneath the druse. Pericytes $(P)$ can be distinguished by their plasmalamellal densities and unusually voluminous cytoplasm, but serial sections suggested the cell process in the eroded segment belonged to a macrophage. Note the response seems to be directed focally towards a druse despite the fact that these drusen may be less than half the height of the RPE and not visible clinically. $E=$ endothelial cell. $L=$ Leucocyte in lumen $\times 5,040$

migrated towards this defect (Figure 8). Cell processes were invading Bruch's membrane and a nearby macrophage contained material apparently removed from the membrane (Figures 9,10). These thinned segments were 40-80 microns wide but the deepest penetration occurred beneath hard drusen. These drusen were sometimes very small, less than half the height of a pigment epithelial cell and so would not have been noted clinically.

Case 4. AMD with small hard and soft confluent drusen, complicated by early subretinal neovascularization (SRN).

Figure 11 illustrates apparent erosion of Bruch's membrane from the eye of a 75-yearold man whose other eye had developed a recent disciform scar. There were multiple such thinned segments up to 150 microns in length, indicating that when the membranous debris was widespread as in this eye, the macrophage response also occurred over a wide area (Figure 12). This attentuated seg- ment lay beneath soft drusen but the material from a hard druse was also present (Figure 13) and the thinning was again most evident beneath small hard drusen.

A distended cell process was observed inside a druse (Figure 13) similar to those illustrated in Figures 3 and 4, but in addition collagen fibrils derived from an adjacent fibroblast were traced passing through the fragmented elastic lamina in this region. The disruption of Bruch's membrane was therefore more advanced in this eye and a small new vessel was found closer to the centre of the fovea.

\section{Discussion}

It has not been established that the debris that accumulates in Bruch's membrane acts as an indirect angiogenic factor $^{7}$ by attracting macrophages from the choroid, but a relationship can be shown between the different forms of debris and the stages of disease.

Most of the material found in Bruch's membrane after the second decade is derived from the rupture of membrane-bound bags believed to represent cytoplasmic material discarded by the RPE. ${ }^{8.9}$ These bags are enclosed by a dense limiting membrane coated on both sides of a lipid bilayer. On rupture they release their content of predominantly amorphous material, together with fragments of the coated membrane. These components accumulate mainly in the outer collagenous zone which consequently thickens much more then the inner collagenous zone. ${ }^{10}$ The bags and spilled contents that remain in the inner collagenous zone become incorporated into small hard drusen.

The present study has shown cellular activity occurring at both these sites. Prior to the onset of AMD as defined earlier, hard drusen are well tolerated for many years but may eventually be cleared by cells that may be RPE derived. Once AMD develops a macrophage response from the choroid is observed in relation to the outer collagenous zone.

The BLD can be regarded as another form of debris. By light microscopy this was the hallmark of $\mathrm{AMD}^{5}$ and was maximally developed at the stage when SRN occurs. However, it also persists for many years within an area of geographic atrophy ${ }^{11}$ and in this situ- 


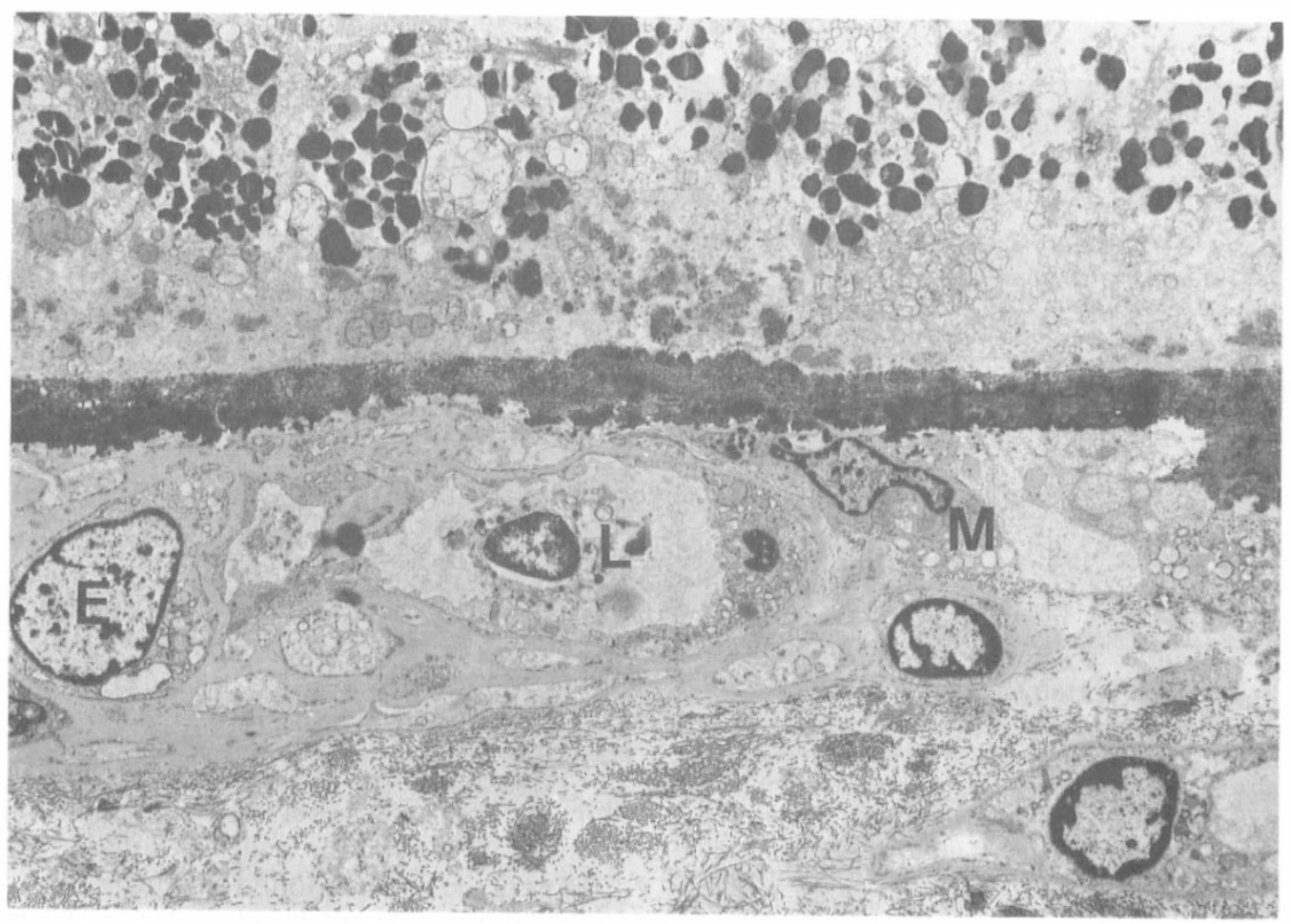

Fig. 9. (Case 3) Serial section of the same vessel shown in Figure 8. The outer collagenous zone is thinned over a wide area. A macrophage $(M)$ is insinuating itself between the outer collagenous zone and the underlying capillary. $E=$ endothelial cell. $L=$ Leucocyte in lumen. $\times 3,130$

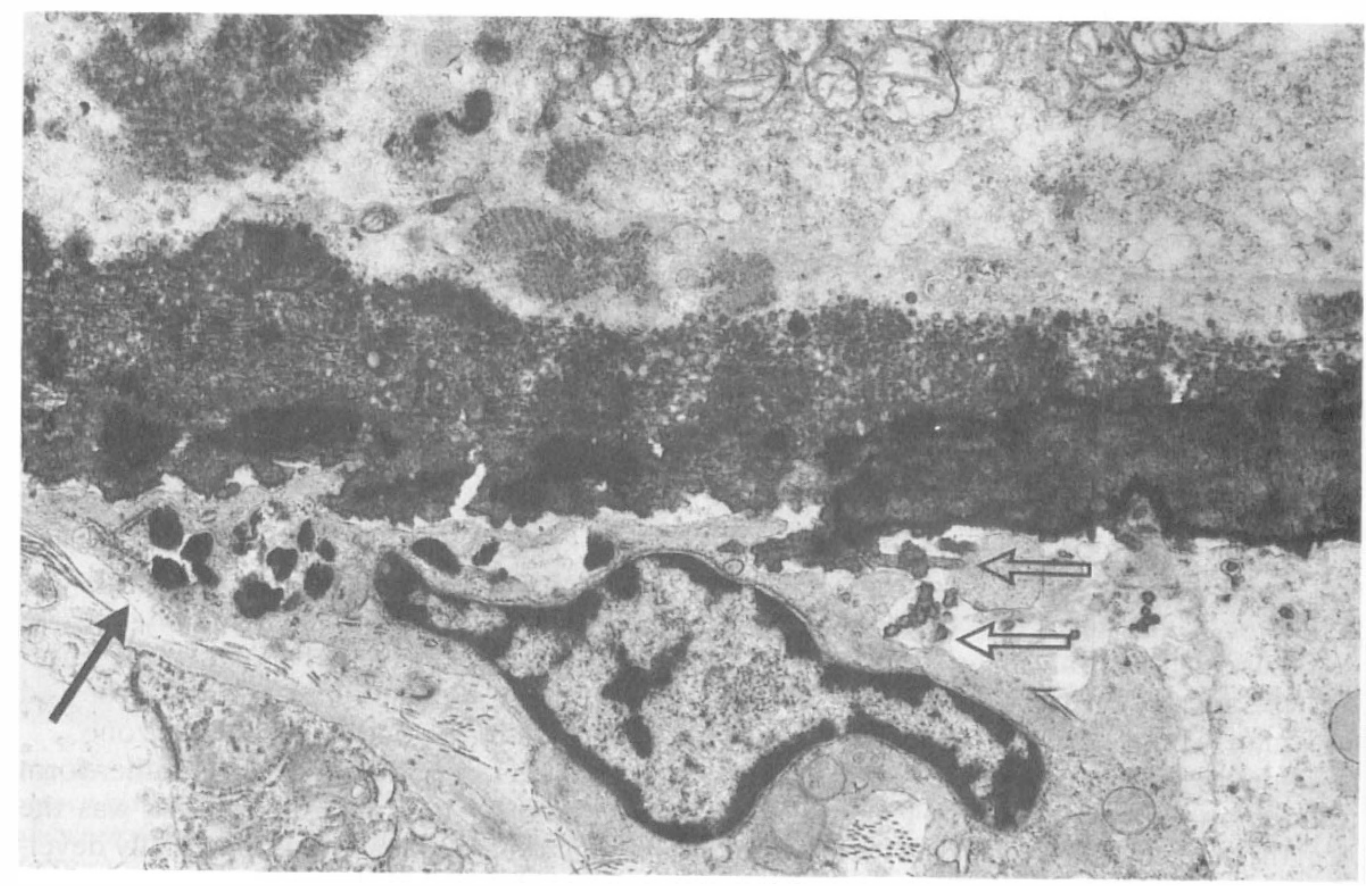

Fig. 10. (Case 3) Higher magnification of the macrophage in Figure 9, showing cell processes enveloping dense fragments of the outer collagenous zone (white arrows). Similar material appears to have been internalised (black arrow). $\times 11,000$ 


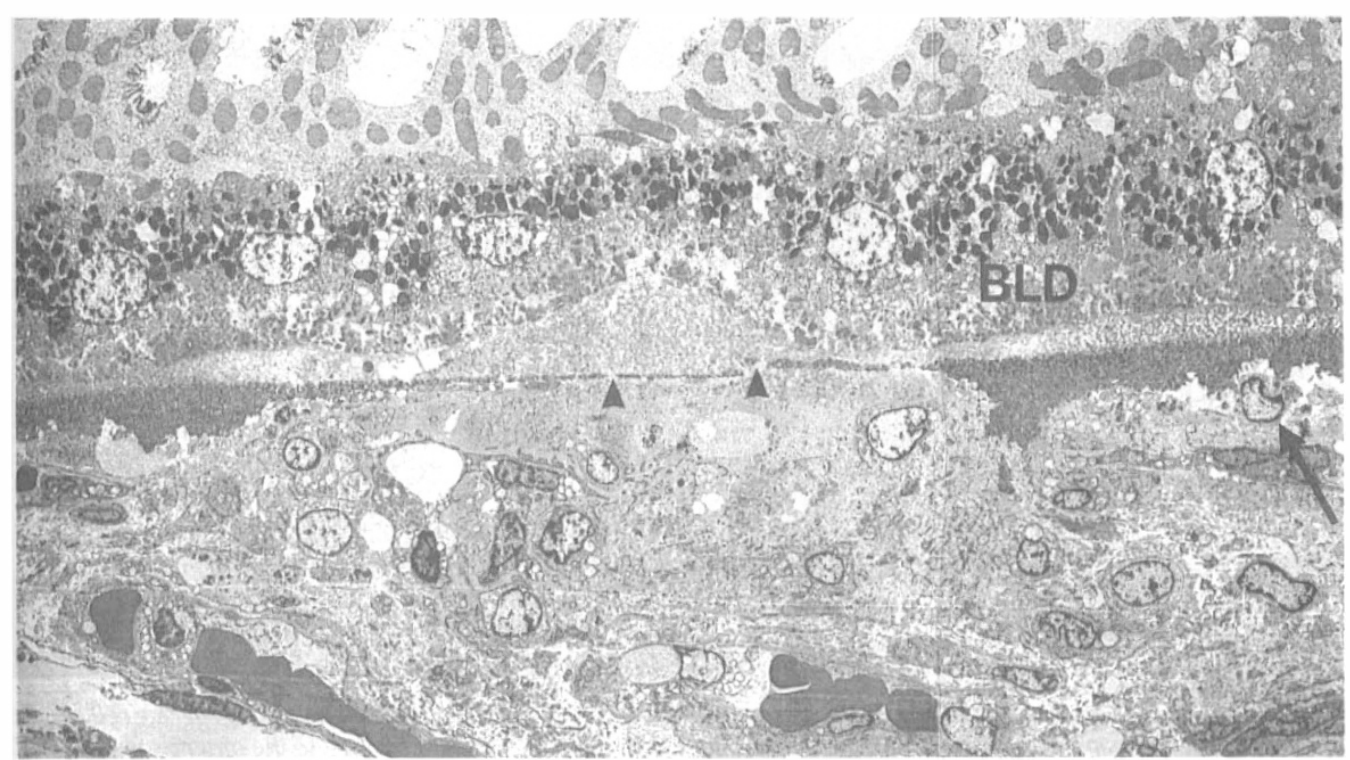

Fig. 11. Case 4 is a patient who had been observed to develop soft drusen in both eyes over a 10-year period. The other eye was noted to have a haemorrhagic disciform scar two months before death at age 75. Section shows a continuous layer of membranous debris under the BLD. The denser mound in the centre consists of collagen fibres, beneath which a fragmented elastic layer is all that remains of Bruch's membrane (arrowheads). The underlying choroidal capillaries are absent and there is a cellular infiltration of the choroid. Cells are also related to the outer collagenous zone beyond the eroded segment (arrow). $\times 1,650$

ation it appears inert. Ultrastructural studies have thrown more suspicion on the coiled membranes that are found under the basal plasma membrane of the RPE and as a layer

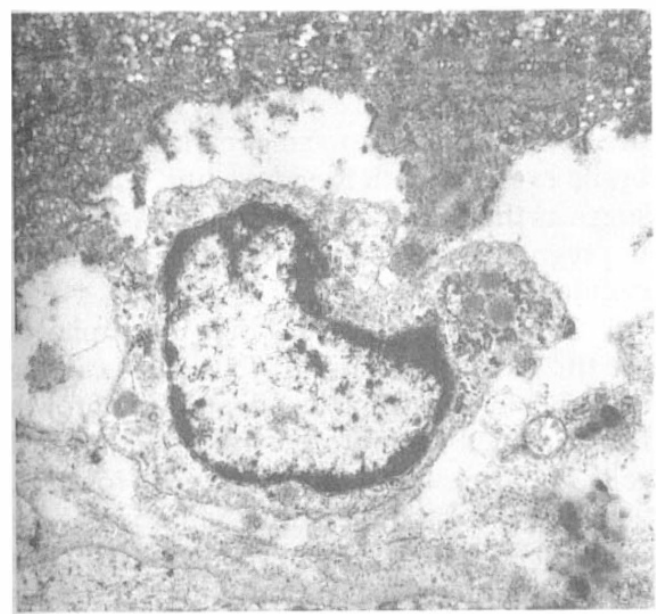

Fig. 12. (Case 4) The cell arrowed in Figure 11 is a macrophage containing lysosomes. It is enveloping fibrous banded material on the external aspect of the outer collagenous zone. $\times 15,500$ of variable thickness external to the basement membrane. ${ }^{11}$ This membranous material has the bilayered structure of plasma membrane and the bulk of the membrane lipid is presumably phospholipid and cholesterol. It is the main ultrastructural constituent of soft drusen and can also be traced into Bruch's membrane. When it was widespread the cellular mobilisation in relation to the outer collagenous zone was more extensive.

The fact that thinning of the membrane was often most advanced beneath hard drusen may be due to the combination of phospholipid in the membranous debris together with neutral fat in the druse and collagenous zones. Histochemical staining on frozen sections of Bruch's membrane confirms the build up of both neutral fats and phospholipids with age ${ }^{12}$ and a focal concentration of these materials may produce a more powerful chemotactic stimulus, possibly acting via a complement cascade.

Choroidal capillaries occasionally appear to migrate into these thinned sections of Bruch's membrane, suggesting these changes 


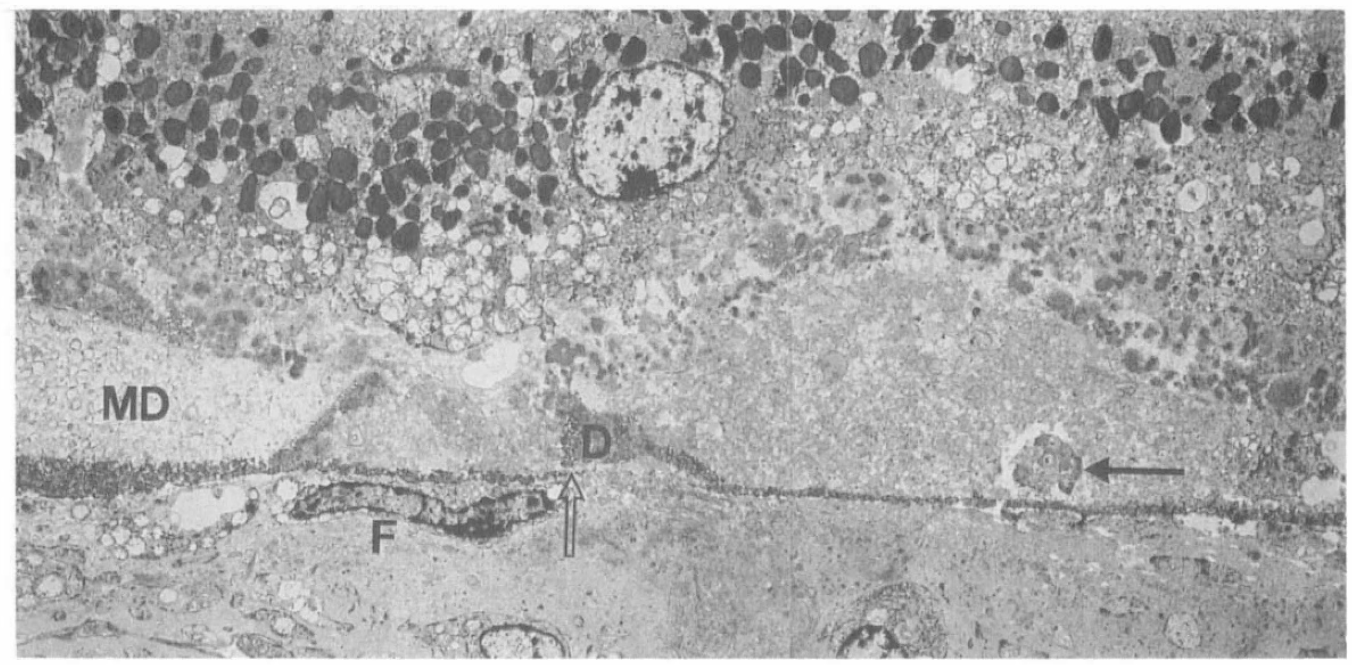

Fig. 13. (Case 4) Serial section through the same eroded segment of Bruch's membrane. A degenerate cell process lies on the internal aspect of the membrane (black arrow) and a fibroblast $(F)$ is applied to the outer aspect. Bruch's membrane is extremely attenuated over a distance of 100 microns and collagen fibrils pass through a microbreak (white arrow). Membranous debris (MD) is raised into soft drusen and over the break there appears to be the remains of a small hard druse $(D)$. This eye also contained a subfoveal neovascular membrane passing through a different part of the membrane. $\times 3,500$

precede SRN. Eyes with established new vessels also demonstrate thinning of Bruch's membrane for some distance around the break and cell processes or collagen fibrils may be found passing through other attenuated segments. This activity is associated with a deeper choroidal infiltration comprising macrophages, lymphocytes, fibroblasts and myofibroblasts.

Activated macrophages secrete enzymes such as collagenase and elastase into the extracellular environment. ${ }^{13}$ The present study suggests these cells are capable of causing erosion of Bruch's membrane by a combination of mechanical disruption, phagocytosis and extracellular release of enzymes. This is consistent with the finding of Zhu et al. ${ }^{14}$ that macrophages had penetrated Bruch's membrane following the subretinal injection of autologous vitreous in rabbits.

However, more than one cell type may be involved in the disruption of Bruch's membrane. In experiments designed to cause resolution of drusen with low intensity laser burns, pericytes have been shown capable of extending processes through Bruch's membrane as part of a phagocytic response. ${ }^{15}$ Moreover, the application of krypton burns not intense enough to destroy Bruch's mem- brane ${ }^{16}$ have established that endothelial cells can likewise invade Bruch's membrane without a preformed break. In these experiments the stimulus for endothelial budding was probably an initial polymorphonuclear leukocyte infiltration that was in turn followed by macrophages. This differs from the natural course of AMD in the human where an acute phase of inflammation is not seen and only chronic inflammatory cells, particularly macrophage-type cells, are found. ${ }^{1}$ In AMD the macrophages are probably attracted to debris, continuing to remove Bruch's membrane even beneath long standing scars. This suggests that once the membrane has become impregnated with lipid it remains a target for cellular attack.

Pollak et al. ${ }^{17}$ suggested that macrophages are the factor common to all diseases causing SRN. They are capable of secreting growth factors which promote growth of endothelial cells, pericytes and fibroblasts. ${ }^{18}$ However, new vessel formation is now regarded as the result of an imbalance between an expanding group of stimulating or inhibiting substances which Glaser ${ }^{19}$ termed extracellular modulating factors. Most attention has been directed to the RPE as the source of these factors, but these may also act indirectly by attracting 
macrophages. Macrophages may be one of the links in the chain of events leading to subretinal neovascularisation.

\section{References}

${ }^{1}$ Sarks S, Penfold P, Killingsworth M, Moussa A. Drusen and their relationship to choroidal neovascularization. In Henkind $P$ ed. Acta: XXIV International Congress of Ophthalmology. Philadelphia, JB Lippincott Co, 1983; 401-404.

${ }^{2}$ Penfold PL, Killingsworth MC, Sarks SH. Senile macular degeneration: The involvement of immunocompetent cells. Graefes Arch Clin Exp Ophthalmol 1985, 223: 69-76.

${ }^{3}$ Korte GE, Reppucci V, Henkind P. RPE destruction causes choriocapillary atrophy. Invest Ophthalmol Vis Sci 1984, 25: 1135-45.

${ }^{4}$ Weiter $\mathbf{J}$ and Fine BS. A histologic study of regional choroidal dystrophy. Am J Ophthalmol 1977, 83: 741-50.

${ }^{5}$ Sarks SH. Ageing and degeneration in the macular region: a clinicopathological study. Br J Ophthalmol 1976, 60: 324-41.

${ }^{6}$ Gass JMD. Stereoscopic atlas of macular diseases: diagnosis and treatment. St. Louis, The CV Mosby Co. 1987, pp. 60-67.

${ }^{7}$ Folkman $\mathbf{J}$ and Haudenschild C. Angiogenesis by capillary endothelial cells in culture. Trans $\mathrm{Oph}$ thalmol Soc UK 1980, 100: 346-53.

${ }^{8}$ Feeney-Burns L and Ellersieck MR. Age-related changes in the ultrastructure of Bruch's membrane. Am J Ophthalmol 1985, 100: 686-97.

${ }^{9}$ Ishibashi T, Sorgente N, Patterson R, Ryan SJ. Ageing changes in Bruch's membrane of monkeys: an electron microscopic study. Ophthalmologica 1986, 192: 179-90.

${ }^{10}$ Killingsworth MC. Age-related components of
Bruch's membrane in the human eye. Graefes Arch Clin Exp Ophthalmol 1987, 225: 406-12.

${ }^{11}$ Sarks JP, Sarks SH, Killingsworth MC. Evolution of geographic atrophy of the retinal pigment epithelium. Eye 1988, 2: 552-77.

${ }^{12}$ Pauleikhoff D, Harper CA, Marshall J, Bird AC. Ageing changes in Bruch's membrane: a histochemical and morphological study. Ophthalmology, (in press).

${ }^{13}$ Jones PA and Werb Z. Degradation of connective tissue matrices by macrophages. II. Influence of matrix composition on proteolysis of glycoproteins, elastin and collagen by macrophages in culture. J Exp Med 1980, 152: 1527-36.

${ }^{14}$ Zhu Z, Goodnight R, Ishibashi T, Sorgente N, Ogden TE, RyanSJ. Breakdown of Bruch's membrane after subretinal injection of vitreous. Role of cellular processes. Ophthalmology 1988, 95: 925-9.

${ }^{15}$ Duvall $\mathbf{J}$ and Tso MOM. Cellular mechanisms of resolution of drusen after laser photocoagulation. An experimental study. Arch Ophthalmol 1985, 103: 694-703.

${ }^{16}$ Pollak A, Heriot WJ, Henkind P. Cellular processes causing defects in Bruch's membrane following krypton laser photocoagulation. Ophthalmol 1986, 93: 1113-19.

${ }^{17}$ Pollak A, Korte GE, Heriot WJ, Henkind P. Ultrastructure of Bruch's membrane after krypton laser photocoagulation. II. Repair of Bruch's membrane and the role of macrophages. Arch Ophthalmol 1986, 104: 1377-82.

${ }^{18}$ Polverini PJ, Cotran RS, Gimbrone MA Jr, Unanue ER. Activated macrophages induce vascular proiferation. Nature 1977, 269: 804-6.

${ }^{19}$ Glaser BM. Extracellular modulating factors and the control of intraocular neovascularization. Special Article. Arch Ophthalmol 1988, 106: 603-607. 